\title{
Modelling and design of a two axis small scale solar tracking system for an ecological small scale house model
}

\author{
Philippe Dondon ${ }^{1, *}$, Pascal Gauterie ${ }^{2}$, Renaud Charlet $^{3}$, \\ ${ }^{1}$ ENSEIRB MATMECA - Bordeaux INP, Domaine Universitaire 33405 TALENCE, France \\ ${ }^{2}$ Lycée Alfred Kastler, 14 av. de l'Université 33402 Talence, France \\ ${ }^{3}$ IMS, UMR 5218, EPHE, 351 cours de la Libération 33405 Talence Cedex
}

\begin{abstract}
Nowadays power generation is one of the greatest challenges of humanity in the framework of Sustainable Development. For example, as it is globally accepted sun tracking systems allows improvement of solar panel power ratio. In order to illustrate this concept, this paper presents the design and a behaviour modelling of a two axis small scale system for future didactical applications. The principle of tracking is described. Mathematical description is done and a mixed SPICE modelling of the system, including geometrical, optical, electronic linear and non-linear aspects is built. Simulations results are analysed. Practical mechanical and electronic designs are detailed, before conclusion. This small scale solar tracking system is now installed in a eco-friendly small scale house model.
\end{abstract}

\section{Introduction}

\subsection{Small scale house project}

Three years were necessary to design a functional realistic eco-friendly small scale house, built in genuine materials. It was completed successfully within the framework of an innovative sustainable development project. The building (with real materials) of small scale house itself is finished [1]. The model is now used as:

- Demonstrator (sustainable development exhibition in town halls or local sustainable development events)

- Didactical support for practical lessons and electronic projects, for sensitizing engineering students to power saving and low power electronic design in first and 2 nd year study.

To make the house model more realistic, didactical and functional, various accessories and their electronic control circuits were designed, for example:

- Low power LED lighting for the terrace and house supplied by a roof solar panel and battery cells.

- Hydrogen fuel stack to power "air conditioned" system (i.e. scaled Canadian well under the house),

- A solar dish with temperature measurement,

- A solar tower and its performance measurement system,

- An electrical heater circuit for the house and its temperature control

- Solar tracking system for small solar panel $(9 \mathrm{~cm} \mathrm{x}$ $5 \mathrm{~cm}) \ldots$

Figure 1 shows a picture of the finished modular scale model $(1 / 20$ scale, i.e. house is $50 \mathrm{~cm} \times 50 \mathrm{~cm}$, garden $100 \mathrm{~cm} \times 120 \mathrm{~cm})$.

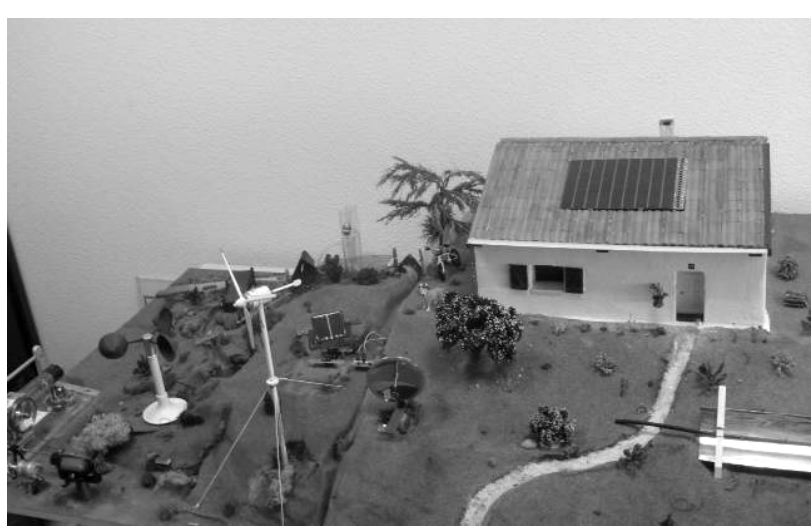

Fig. 1. View of small scale house and surroundings.

The present paper focuses on the modelling and design of the small scale 2 axis solar tracking system installed in the "house garden". This new system is an improved version of a first previous design which was working only on one axis tracking [2].

\subsection{Solar tracking interest}

Professional and industrial true systems are generally based on processor board; the position of the sun is generally tabulated (from astronomical data), all over the year. Position of the solar panel follows the programmed law on two axes, taking into account the sun ray optical diffraction or refraction into atmosphere. From experiments and available data [3, 4], a real solar tracking system can improve the electricity production of 
a solar panel up to $23 \%$ for a single axis tracker and $27 \%$ for a double axis tracker.

\section{Solar tracking circuit}

\subsection{Generalities}

The designed circuit, mostly based on analogue electronic components, is obviously a simplified version compared to a true tracking system $[4,5]$. However, it is a complex looped system which requires a full modelling to predict behaviour, accuracy and stability, before electronic design.

\subsection{Tracking system principles and schematic}

As the "eco-friendly house" is used for in-door demonstration, a halogen spot light will "replace" the sun light.

The design is based on a simple system with two rotation axis control. Figure 2 shows the sun tracking block diagram.

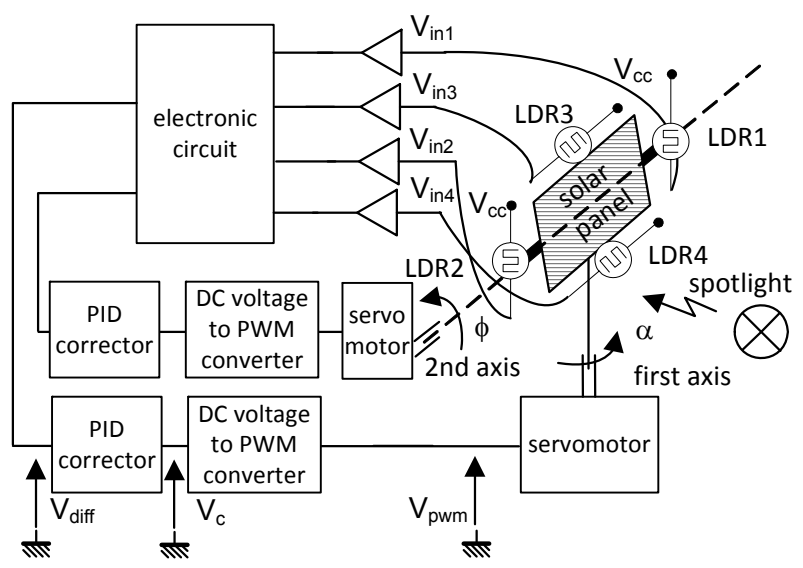

Fig. 2. Sun tracking system block diagram

The same principle is used on the two axis (elevation $\phi$ and azimuth $\alpha$ ) for the feedback control loop; this explanation is given only for the azimuth control.

It works as follows: two light sensors LDR1 and LDR2 (LDR: Light Dependant Resistors), located on left and right side of the panel receive the "sun" light.

When the solar panel is well align in "sun" direction, the received left and right lights flux L1 and L2 are equal. When it is not, one of the two LDR receives more flux than the other, and feedback loop moves the servo motor into the right direction to cancel the voltage difference $\mathrm{V}_{\text {diff }}$ "left minus right" signals (Cf. figure 3 ). $\mathrm{V}_{\text {diff }}$ being a DC voltage, a DC to PWM converter is lastly required: indeed, the servomotor is a classical hobbyist servo. The rotation angle of such servomotor is proportional to a control signal pulse width $\mathrm{V}_{\text {pwm }}\left(1 \mathrm{~ms}\right.$ to $2 \mathrm{~ms}$ for a $180^{\circ}$ range) according to hobbyist standards.

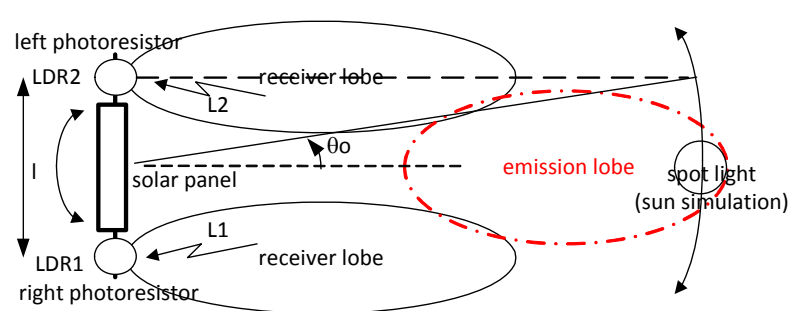

Fig. 3. Solar panel alignment

\subsection{Modelling interest}

The principle explained in $\$ 2.2$ is well known by electronic designers. Similar applications using infrared diodes or ultrasonic sensors instead of LDR are described in [6-9]. Thus, an "intuitive" electronic design can be directly done without a preliminary high level theoretical approach.

However, for a fine understanding of parameters impact (i.e. spotlight distance, matching and non-linearity of LDR sensors, mechanical behaviour of servomotor etc.), a global modelling is required as described in $\S 3$.

\section{Tracking system modelling}

\subsection{One axis feedback loop modelling}

One could note that modelling is not easy because the whole system consists of several heterogeneous parts. That requires a global approach.

Among several possibilities, (analytic calculus, MATLAB software, SPICE simulator...) we propose hereafter, the building of a mixed SPICE modelling adapted to our electronic initial culture.

It must take into account the electronic behaviour (Electronic circuits, non-linear sensors (LDR1 and LDR2), the mechanical behaviour (servomotor), and also the optical and geometrical aspects, due to the rotation of the spot light (simulating the sun).

Preliminary step is to establish a block diagram representing the global feedback system.

In order to simplify the approach, the range of rotation angle -i.e. $180^{\circ}$ for a classical servomotor- is supposed covering a sweep from East to West.

Figure 4 shows the identified blocks of the whole system and figure 5 gives the corresponding geometrical angles for one rotation axis $\alpha$ (azimuth).

- $\mathrm{L}_{1}, \mathrm{~L}_{2}$ represents the received light (in Lux) on each LDR,

- $\alpha_{\text {ref }}$ is the main input and represents the sun azimuth position $\left(0^{\circ}\right.$ to $\left.180^{\circ}\right)$,

- $\alpha$ the angle given by the servo motor,

- $\alpha_{\mathrm{e}}$ the error angle,

- Source light intensity input (I) can be seen as a parasitic input in the loop which represents fluctuation of source intensity (fog, sky partially cloudy, etc),

- $\Theta_{0}$ is the angle defined as $\Theta_{0}=\operatorname{arctg}(l / 2 \mathrm{D})$, where $l$ is the distance between sensors and $\mathrm{D}$ the distance to spotlight as shown on figure 3 , 
- Local disturbances input (D) represent disturbances that can occur individually, on each LDR optical way. (LDR partially hidden by an object passing over, etc).

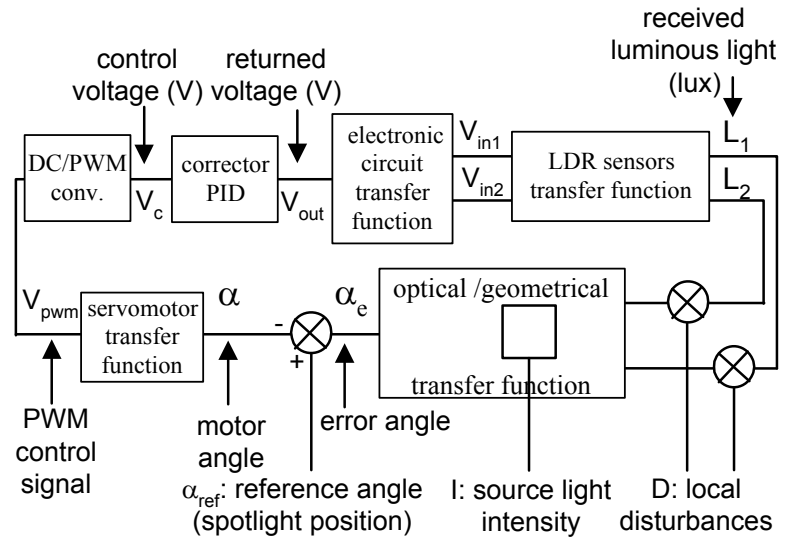

Fig. 4. System identification

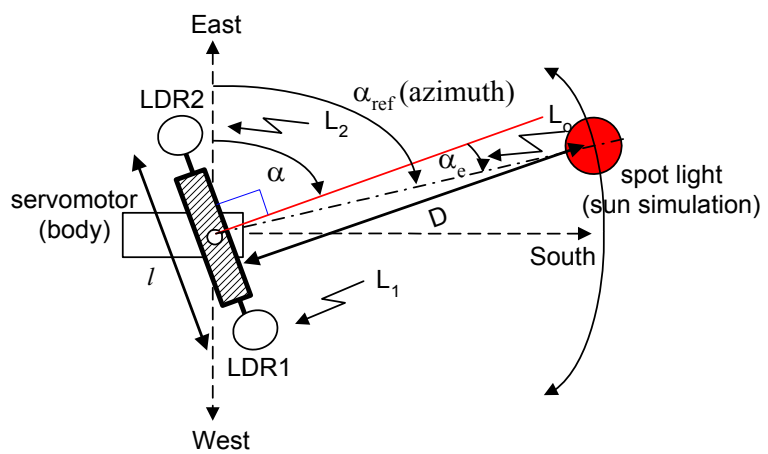

Fig. 5. 2D reference angles (top view)

Feedback loop for the second rotation axis is similar. And the two axis movements are obviously coupled to track the spot light. Thus, coupling equation between elevation and Azimuth must be known (cf. §3.2).

\subsection{Sun position modelling}

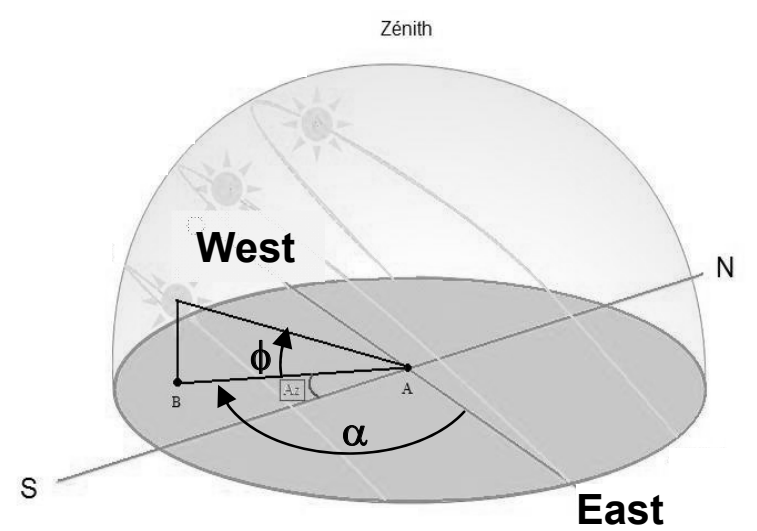

Fig. 6. $3 \mathrm{D}$ azimuth and elevation diagram

In figure 6 , it is depicted a $3 \mathrm{D}$ representation of two angles $\alpha$ and $\phi$.

From the web site "sunearthtools.com" [10] and Google maps, one can obtain the sun position everywhere on planet in real time. For example, figure 7 gives the situation for Bordeaux Talence University, the 21 of June 2017 (summer solstice).

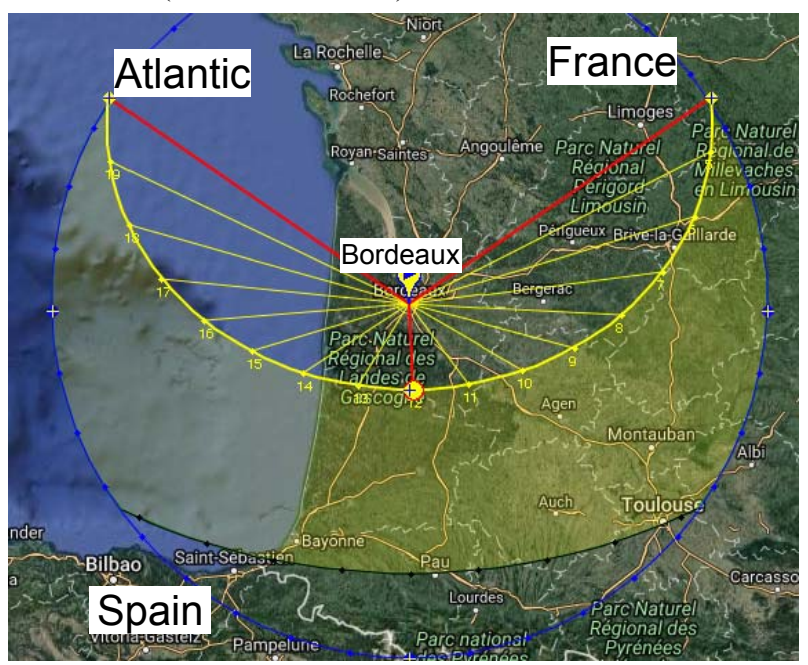

Fig. 7. Sun path over Bordeaux city (hours and direction).

Figure 8 shows the corresponding sun elevation angle $\phi\left({ }^{\circ}\right)$ vs. azimuth angle $\alpha\left(^{\circ}\right)$. Lower curve corresponds to winter solstice, and upper curve to summer solstice.

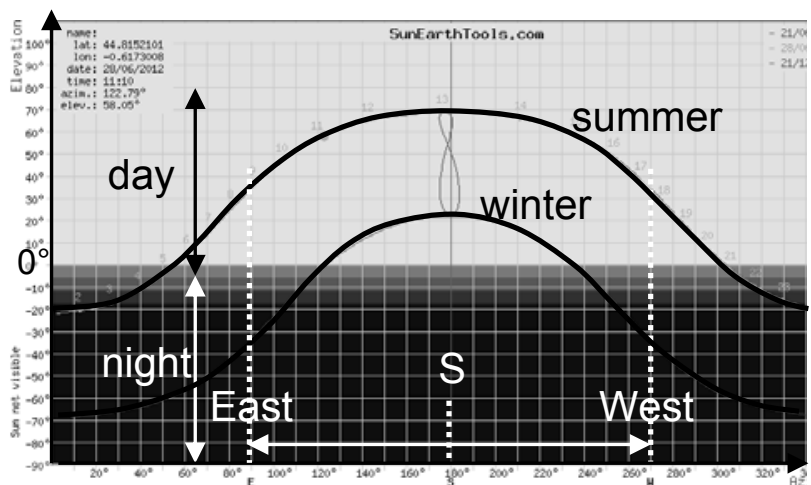

Fig. 8. Sun elevation vs. azimuth (in ${ }^{\circ}$ ) in Bordeaux

Horizontal scale: azimuth $0^{\circ}$ to $360^{\circ}$ (south $<=180^{\circ}$ ) Vertical scale: elevation $-90^{\circ}$ to $+90^{\circ}$

From figure 8 , we can extract a mathematical $2^{\text {nd }}$ degree function (figure 9) to approximate theses curves on the interesting segment (i.e. during the day, East to West).

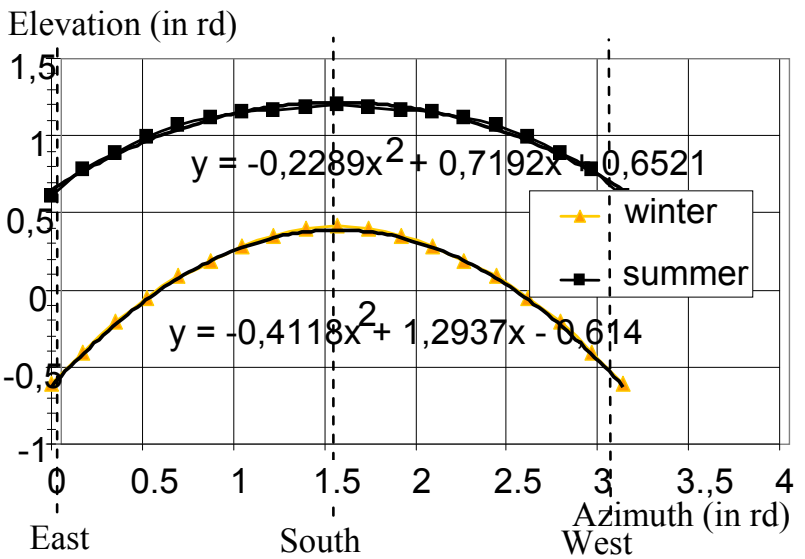

Fig. 9. Elevation modelling in Bordeaux (in rd) 
Between these two curves, we can extrapolate an average annual behaviour.

We can write:

$$
\phi \#-0.3 \alpha^{2}+0.945 \alpha+0.1 \quad \text { (angles in rd) }
$$

\subsection{Electronic modelling}

The main parts of electronics feedback loop circuits (LDR sensors nonlinear transfer function, analogue subtraction circuit...) have been previously modelled. Details can be found in [2].

\subsection{Optical/ Geometrical modelling}

The mathematical function "received light $\mathrm{L}_{1}$ and $\mathrm{L}_{2}$ (in lux) on each LDR sensors versus incidence angle of the spotlight" is also required. Fluxes vary roughly as the cosines of incidence angle. They depend on the spot light distance and also on the distance between LDR sensors. Details are also given in [2].

\subsection{Modelling of servomotor}

Classical Hobbyist servo Hitec or equivalent, medium sizes are used. Supply voltage is $+5 \mathrm{~V}$. The control signal Vpwm is a PWM periodic signal: period $20 \mathrm{~ms}$, pulse width $1 \mathrm{~ms}$ to $2 \mathrm{~ms}$ for an angle range of $180^{\circ}$. Maximum rotation speed is $60 \% / 0.15 \mathrm{~s}$. Modelling includes internal feedback loop and rotation speed saturation. Details can also be found in [2].

\subsection{One axis tracking system modelling}

All the blocks were assembled to build a basic one axis equivalent Spice schematic. It has been previously detailed in [2]. After some adaptations, it is usable for the two axis system. The schematic includes all sub blocks discussed in $\S 3.3,3.4,3.5$ and is displayed in figure 10. Since SPICE stimuli are obviously in Volt and Amp, correspondence scales have been defined as follows:

$1 \mathrm{Vdc}<=>1$ Lux for light flux

$1 \mathrm{Vdc}<=>1 \mathrm{rd}$ for angles

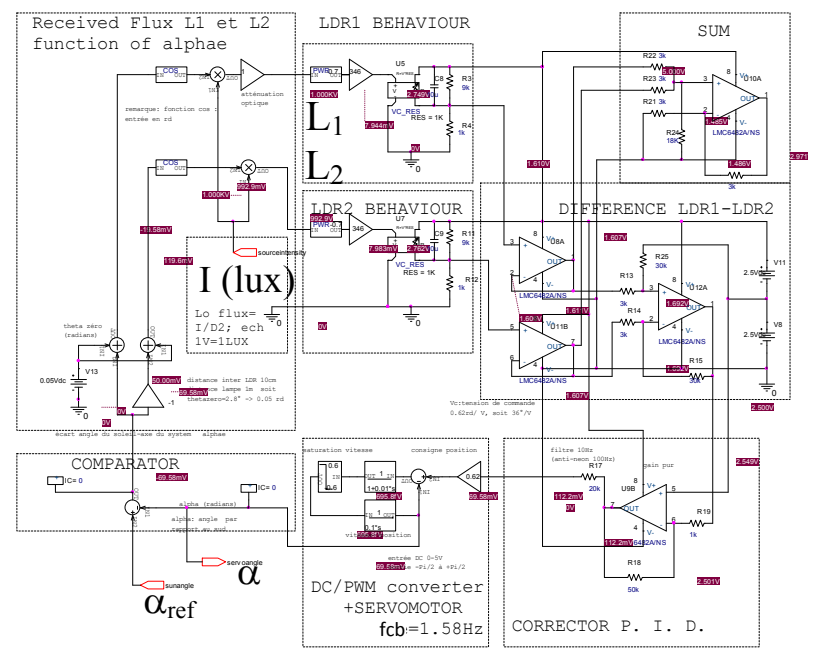

Fig. 10. One axis tracking modelling

\subsection{Two axis modelling}

As the two axis works in the same principle, the previous schematic given in figure 10 , is transformed into a SPICE sub circuit. Then, we can duplicate this subcircuit and associate them: The first one will represent the azimuth movement and the second, the elevation movement. Finally, two axis modelling is given in figure 11.

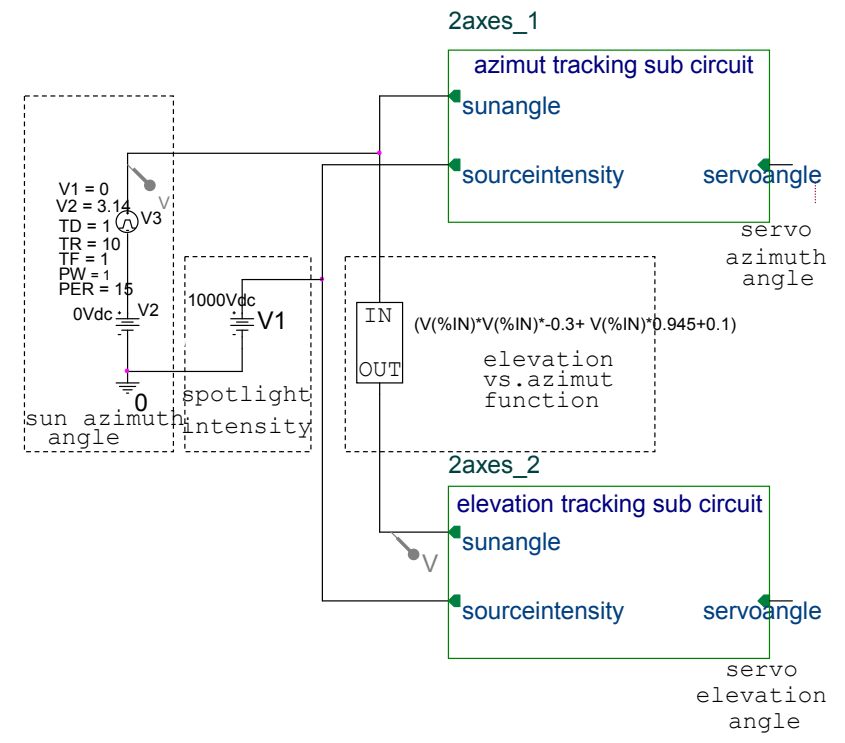

Fig. 11. Two axis solar tracking SPICE modelling

The two sub-circuits are connected together and linked by equation (1) which is represented by an ABM SPICE library block (cf. §3.2). Main inputs are the spotlight azimuth $\alpha$ ref, (represented by a ramp voltage source V3 from 0 to $3.14 \mathrm{~V}$ ), and the spot light intensity $\mathrm{L}$ (represented by a DC voltage source V1). (1000Vdc $\Leftrightarrow$ 1000 Lux).

Mains available modelling outputs are azimuth and elevation angles ( $\alpha$ and $\phi$ ) given by the two servomotors and error angles to check accuracy of the system $\alpha$ e and фe.

\section{Spice simulations}

Among numerous simulations, we give hereafter the most significant ones.

As SPICE works obviously in Volt and Amp, horizontal and vertical scales of the simulated response curves are converted (if necessary) in radians angles and Lux before display, for better understanding.

\subsection{Open loop one axis simulations}

Figure 12 represents a DC sweep on azimuth axis under the following conditions: the spot light is locked in south position and a rotation of azimuth servomotor from East to West $\left(0^{\circ}\right.$ to $\left.180^{\circ}\right)$, is performed. 


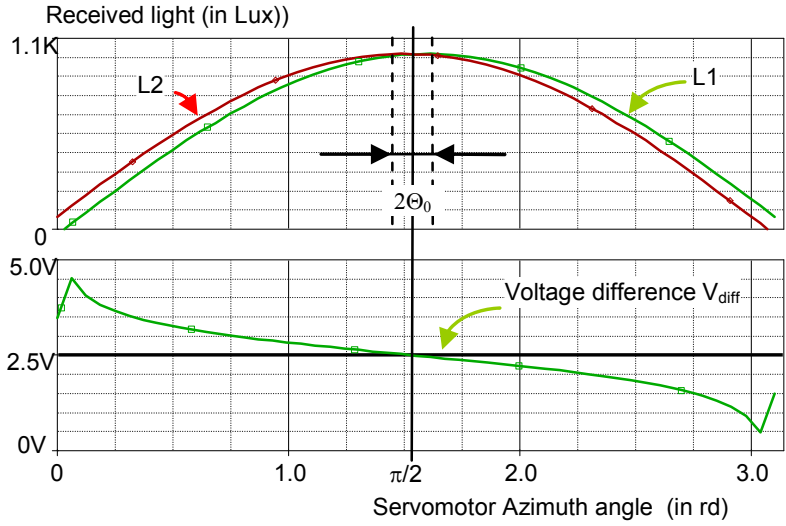

Fig. 12. Open loop simulation (DC sweep)

Upper curves shows $\mathrm{L}_{1}$ (green) and $\mathrm{L}_{2}$ (red) received lights flux on LDR1 and LDR2. $\mathrm{L}_{1}, \mathrm{~L}_{2}$ reach their maximum values $\mathrm{L}_{0}$ (here $=1000$ lux) when the spotlight is exactly in front of each (Respectively for $\alpha=\pi / 2+\Theta_{0}$ and $\left.\alpha=\pi / 2-\Theta_{0}\right)$. Lower curve shows the difference signal $\mathrm{V}_{\text {diff }}$ (as defined in figure 2), centered on Vsupply/2 (i.e. $\mathrm{V}_{\mathrm{cc}} / 2=2.5 \mathrm{~V}$ ). It is a classical " $\mathrm{S}$ " curve: when the spotlight is perfectly aligned, $\mathrm{V}_{\text {diff }}$ is centered on $V_{c c} / 2$. When the source light is shifted above LDR2, $\mathrm{V}_{\text {diff }}$ becomes greater than $\mathrm{V}_{\mathrm{cc}} / 2$. When the source light is shifted above LDR1, $\mathrm{V}_{\text {diff }}$ becomes smaller than $\mathrm{V}_{\mathrm{cc}} / 2$. $\mathrm{V}_{\text {diff }}$ is thus well suitable for a feed-back control. When $\alpha$ reaches 0 or $180^{\circ}$ (Sunset or sunrise situation), L1 and L2 tend together toward zero (tangent light to the LDR surface), thus $\mathrm{V}_{\text {diff }}$ return to $\mathrm{Vcc} / 2$.

Other open and closed loop simulations and experimental characterisation for checking the stability and frequency behaviour of the system were already detailed in [2]. So, they are not given here.

\subsection{Closed loop two axis simulations}

\subsubsection{Response to a full spot light rotation}

A DC sweep is applied on spot light azimuth angle input $\alpha_{\text {ref, }}$, to simulate a full rotation from East to West. Figure 13 shows the corresponding azimuth and elevation servomotors angle response.

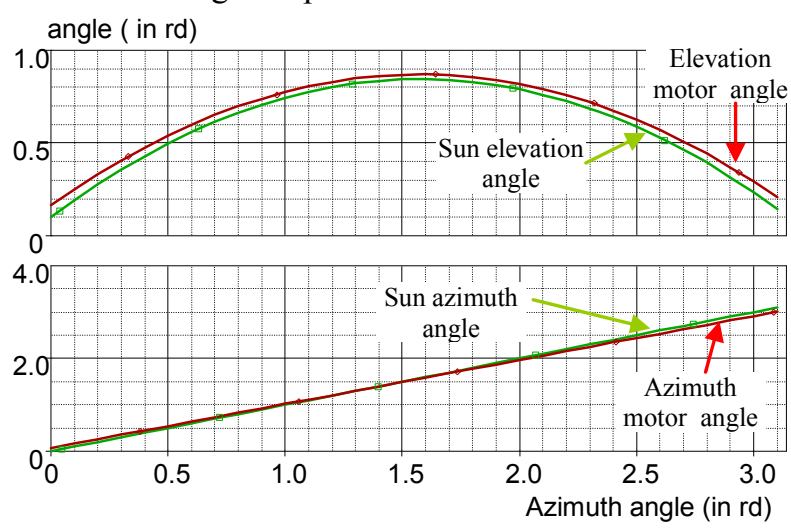

Fig.13. Closed loop, DC sweep response
Upper plot: sun and servomotor elevation angles $\phi e$ and $\phi$ vs. Azimuth angle $\alpha$ ref.

Lower plot: sun and servomotor azimuth angles $\alpha$ ref and $\alpha$ vs. azimuth angle $\alpha$ ref .

Error angles correspond to the difference between green and red curves. Tracking is correct since both error azimuth angle $\alpha_{e}$ and elevation angle remain close to zero (less than $\pm 2.5^{\circ} \mathrm{C}$ ) during all the simulated rotation. However, it must be mentioned that there is no integral correction in the feed-back loop circuits for stability reasons: thus, error angle is proportional to reference angle and slightly increases from East to West as it can be seen on the left side of figure 13 .

\subsubsection{Response to light source intensity fluctuations}

The light source is located quite far (around $50 \mathrm{~cm}$ ) from the LDR sensors, compared to the size of tracking system. Thus, fluctuations of source intensity (I input) should affect identically the four LDRs. They can be considered as a kind of parasitic common mode for the loop. This last simulation (figure 14) is done to check possible effects that can occur due to "in door" uses of the small scale demonstrator (i.e. flicker noise coming from artificial light (fluorescent neon tubes in the room at $100 \mathrm{~Hz}$ ). It shows the transient response when system is submitted to a $20 \%$ fluctuations of the source (200 Lux around de 1000 Lux, square waveform, 10ms period) with spotlight oriented to south.

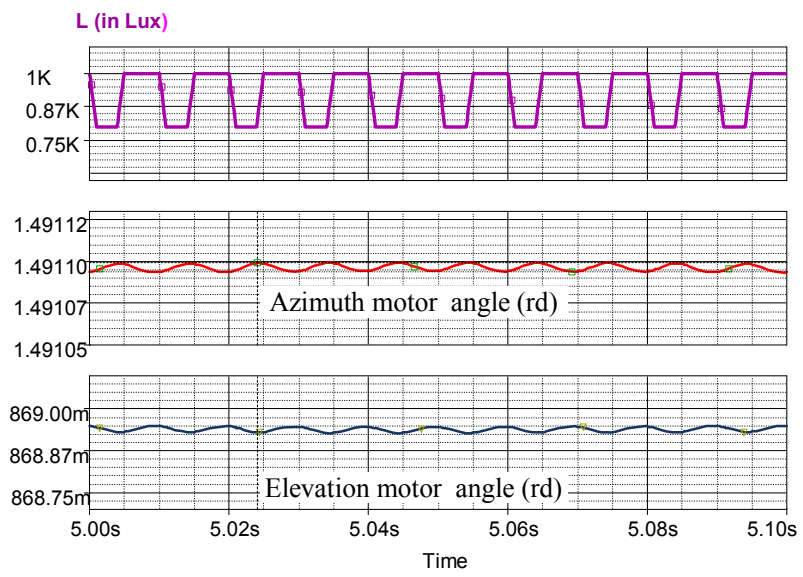

Fig. 14. Closed loop response to fluctuation of source intensity (I input).

Variations around the bias position of azimuth and elevation angles $\alpha$ and $\phi$ are less than $0.1 \mathrm{mrd}$. These parasitic variations are well rejected. Thus simulation confirms an almost total insensitivity of the system while LDR sensors are perfectly matched.

At this opposite, mismatching between the LDR sensors could seriously affect the loop behaviour. Fine impacts and effects have been previously studied in [11].

\section{Practice and measurements}

\subsection{Mechanical assembly}


Figure 15 and 16 shows the installation in the small scale house surroundings. Size of the small solar panel is $9 \mathrm{~cm}$ $\mathrm{x} 5 \mathrm{~cm}$ and electronic board $10 \mathrm{~cm} \times 10 \mathrm{~cm}$.

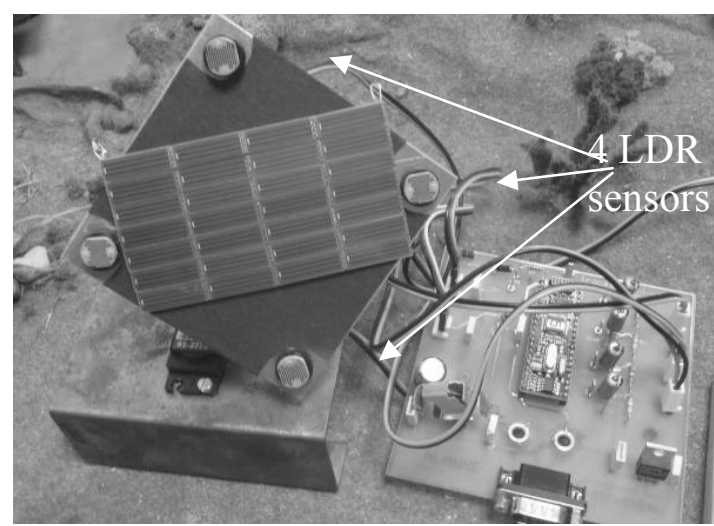

Fig. 15. Small scale solar tracking system (front side view)

\subsection{Electronic design}

The design uses only COTS components. The four matched LDR sensors (VT90N2 series or equivalent) are placed in a Wheatstone bridge. Output signals are subtracted using rail to rail OP amps LMC6482 to have the widest dynamic range. Difference $\mathrm{V}_{\text {diff }}$ is applied to the simple PID corrector (adjustable gain + low pass RC filter). The resulting $\mathrm{DC}$ voltage value is processed by a microcontroller MC9S12C128 module to generate PWM signals for controlling the servos Hitec 322-HD.

Actually, this module was not necessary: a simple analogue to PWM voltage converter should be greatly enough. But we used it because it also processes various data that could be transmitted by CAN bus to a distant computer. Supply voltage comes through a LM7805 regulator $+5 \mathrm{~V}$.

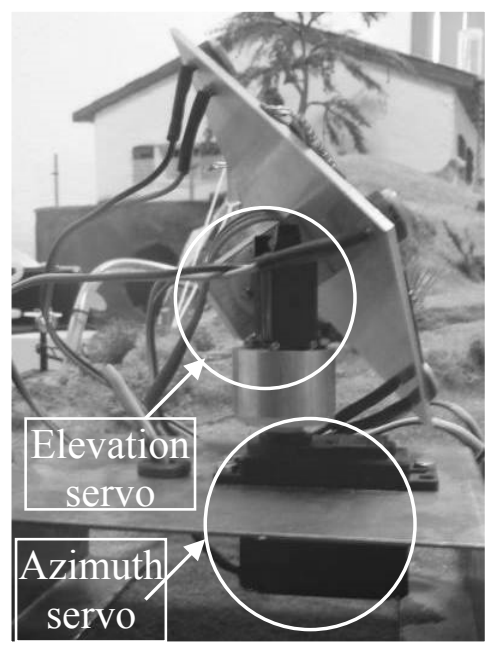

Fig.16. Small scale solar tracking system (back side)

\subsection{Static open loop measurement}

A preliminary open loop characterisation was performed on azimuth axis mainly to check the servomotor transfer function (no manufacturer data). First cut-off frequency occurs at $1.58 \mathrm{~Hz}$ which is quite common for this kind of servo.

Secondly, the spotlight was located to south with zero degrees elevation. The voltage difference LDR1, LDR2 $\mathrm{V}_{\text {diff }}$ vs. azimuth angle was plotted using a simple DC voltmeter. This experimental curve (figure 17) can be easily and successfully compared to the simulated response in figure 12 .

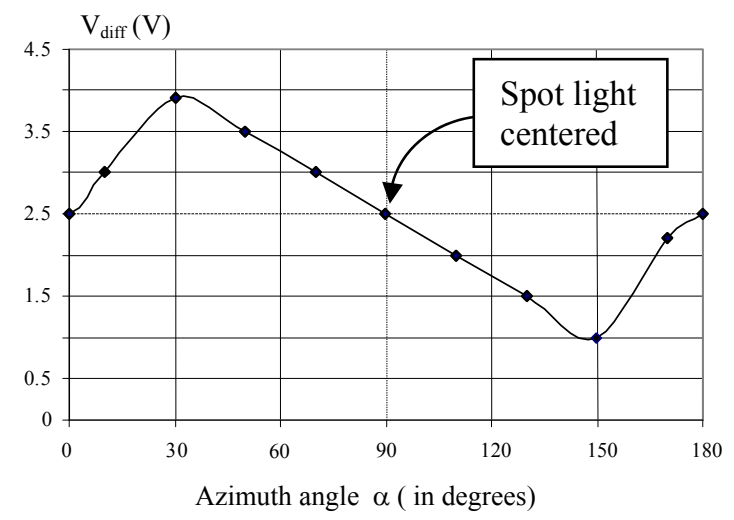

Fig. 17. $V_{\text {diff }}$ vs. azimuth angle $\alpha$

\subsection{Visual test}

Once the system looped, we checked by visual tests that tracking feedback loop worked correctly separately on each axis and then simultaneously on the two axes. Validation of the system behaviour was done under various conditions (light source distance and intensity).

A correct matching between global SPICE modelling, predicting simulations and experimental behaviour design is observed.

Finally, a small video clip was recorded to show the system behaviour.

\subsection{Disturbance effects}

A shutter (simulating an object passing over) was placed above one of the LDRs to simulate a "local disturbance on D input" as define in figure 4. Servomotor first deviates, and then comes back to the initial position when the shutter is removed.

A second "non-realistic" test, which can be seen as a "step response", was done: moving very suddenly the spot light out of the visual field of the LDR sensors. System goes to the mechanical stop (limited by the servo internal stop) and stays in this position until the spotlight comes again in the visual field of sensors. Then, the tracking starts again.

\section{Discussions}

The current Spice modelling gives satisfaction for predicting and analysing the main performances of the small scale solar tracking system. Main tendencies and order of magnitude obtained by simulation are confirmed by experimental test on the small scale system. However, it is not so easy to get extremely precise angles data from the practical tests: some accessories must be added on 
the test bench to improve accuracy of measurements (in particular, needles to point more finely the azimuth and elevation directions)

Main advantages of the modelling are:

- Quick understanding of the tracking system general behaviour,

- Electrical modelling suitable for those who do not have specific knowledge out of the electronic field,

- Fast Spice simulations,

- Enough but not too much complex for didactical uses.

However, there are obviously some limits:

- Approximations have been done to build the modelling: we cannot be specialists of electronic components, optic and astronomy at the same time. In particular, optical modelling blocks might be refined.

- This is not a parametric modelling. Each parameter (geometrical data, servo motor specifications, light intensity etc.) must be adjusted or changed manually if necessary.

\section{Conclusions}

An equivalent modelling of a small scale two axis solar tracking system was presented. Mixed Spice modelling and electronic analogue designs were successfully validated by experimental tests.

This tracking system is now installed in our small scale house modelling. Lastly, from a didactical point of view, this modelling is now used for training the students during the electronic feed-back projects and practical lessons as "a case of study".

\section{References}

1. Ph. Dondon, P. Cassagne, M. Feugas, C.A. Bulucea, D.Rosca, V. Dondon, R. Charlet de Sauvage, WSEAS Trans on Environment and Development, Vol. 7, pp. 225-235 (2011)

2. Ph. Dondon, L.Miron, WSEAS Trans on Circuits and Systems Vol. 13, pp. 454-463, (2014)

3. Exosun company Web site : http://www.exosun.net/en-us/

4. S. Jacques, A. Clenet, Z. Ren, M. Lescieux, A. Schellmanns, T. Jacques, E. Pluvinet, A Caldeira, N Batut, Proc. of CETSIS Conf, Besançon, France, oct 27-29 (2014)

5. P.J. Axaopoulos, K. Moutsopoulos, M.P. Theodoridis, Proc. of the 8th WSEAS International Conference on Engineering Education, Corfu, Greece, July 14-16 (2011)

6. Ph. Dondon, Enseirb-Matmeca web site : http://dondon.vvv.enseirbmatmeca.fr/PIC/autrapplication/Radardepoursuiteet demesureUltrason.htm

7. T. Bendib, B. Barkat, F. Djeffal, N. Hamia et A. Nidhal, Revue des Energies Renouvelables, Vol. 11 pp.523-532 (2008)
8. M.D. Draou, B. Draoui, Revue des Energies Renouvelables Vol. 11 pp.229-238 (2007)

9. P.L. Milea, O. Oltu, M. Dragulinescu, M. Dascalu, Proc. of the WSEAS Int. Conf. on Renewable Energy Sources, Arcachon, France, October 14-16 (2007)

10. On line material available at http://www.sunearthtools.com/ , accessed 30 june 2017

11. Ph. Dondon, L. Miron, Proc. of AFASES Int. Conf. on scientific research and education, Vol.2, pp.321330, Brasov, Romania, May 28-29 (2015) 